\section{ON THE SECOND SOUND AND MURMUR OF THE HEART AND GREAT VESSELS, IN THEIR RELATION TO DIAGNOSIS.}

By Jонn Cockle, M.D., Physician to the City Dispensary, and to the Margaret Street Dispensary for Consumption, etc.

The following contribution has for its object an inquiry into the second sound and murmur of the heart and great vessels, in their relation to diagnosis. It is solely one of practical character ; in some points, not altogether in accordance with the current teaching; and is, consequently open to the fullest dis cussion and criticism. The facts are stated as they occurred to me, together with such inferences as may be considered fairly warranted from the subject matter.

I shall premise a few observations generally respecting the impulse, rhythm, and sounds of the heart, before entering into the question of the special modifications in disease connected with the second sound, etc. The ordinary expression of the heart striking against the chest-wall appears inaccurate, since it is now pretty generally admitted that the heart, in the perfectly normal state, never disconnects itself from the parietes. The conditions necessary for an actual stroke are, therefore, absent. In the language of Charles Robin, "On ne peut frapper ce qu'on touche et l'on ne bat que ce que l'on ne touche pas." The apparent stroke consequently appears to consist simply in elevation of the corresponding soft structures of the chest-wall. This matter, however, is too complex to challenge from me here the arguments in its support. To pass on, then, to the rhythmic action and sounds of the heart, I may state that I have for some time past been engaged in an experi. mental investigation of these phenomena. Taking into consideration the retrograde course of the coronary arteries, and the fact that, during the systole of the ventricles, the blood of the coronary arteries would be prevented penetrating their tissue, it occurred to me to revive the old inquiry as to the time when the nutrient arteries of the heart were filled. I remembered the physical demonstration of Bernouilli, that, if fluid was injected into a tube of given calibre, it would pass with facility into secondary tubes, the direction of which was in harmony with that of the main branch; but if, on the contrary, the direction of the secondary tube was opposed to that of the trunk, not only would the onward current be prevented passing into such diverticulum, but that fluid would actually be drawn up into the latter from a reservoir below.

I applied, I say, at first, this argument to the action of the coronary arteries, and conjectured, from their direction with re spect to the main trunk, that they rnust be filled during aortic systole. Subsequently I found, experimentally, that this chain of reasoning, however sound with regard to fluid traversing empty tubes, would not accurately apply to those already filled. I have consequently thus modified my view with respect to these phenomena.

While admitting fully a controlling influence of the nervous ganglia of the heart, I adopt, at the risk of being thought antiquated in my notions, the Hallerian doctrine relative to the automatic action of this organ; and contend that, with reference to the invariable sequence between the systole and diastole, it is purely one of muscular action conditioned upon the contact of the blood with its tissue.

I conceive that the heart's alternating systolic and diastolic action is, in the healthy subject, maintained by two causes; the one intrinsic-the contact of blood with the endocardial nem brane; the other extrinsic-the blood injected by the coronary arteries into the proper tissue of the muscle. The necessity of the first cause-the ingress of blood from the cavæ into the auricle-seems proved by the early experiments of Bartholini, and their subsequent modification by Haller, Cardan, Senac, and others. 'The second cause I attempt to explain in the following manner, commencing from that period of a revolution of the heart's rhythm corresponding to the ventricular systole. At this time the auricle is dilating from the blood of the cavæ; its tissue is lax, and easily permeated by aortic arterial blood. These two acts, being simultaneous, determine at once its contraction. The contracted condition of the ventricle during this period had prevented the entrance into its tissue of the nutrient blood; but now, commencing its diastole, and filling with the blood from the auricle, the obstacle is removed, and instant contraction again ensues, as before described.

With regard to the sounds originating at the cardiac orifices, I accept the teaching of Skoda. that there are two engendered at each venous, and two at each arterial ostium.
I incline, however, to the belief that the first ventricular sound may be produced in the following manner. During the closure of the auriculo-ventricular valves, they carry, in the funnel shape partially assumed during the process, a portion of the ventricular blood back into the auricle: this layer is reinforced by blood streaming from the cavæ; so that the reaction between the lower and upper columns, the valves intervening, gives rise to the dull prolonged first ventricular sound, the upper blood-layer acting as a damper to the valve-sound. And this view leads me to consider that, during the ventricular systole, the chamber cannot be entirely emptied. If, during the period of ventricular contraction, we imagine the blood-stream divided into two columns, one rushing through the aortic mouth, the other assisting in the momentarily sustained closure of the auriculo-ventricular valves, this latter portion must, theoretically, hy the nature of the contraction, be left in contact with the ventricular face of those valves, and rendering their mid portions of dome-like form, which latter condition has been recently experimentally demonstrated by Chauveau.

However this may be, one point appears clear, if the recent experiments of Dr. Halford may be considered trustworthy, that the obstruction of the blood from the cave into the heart annihilates the normal sounds. Some indefinite sound, it appears, may still exist; muscular bruit, the visible vibrating motion described by Haller during contraction, caused possibly by the stimulus of the blood, still existing in the coronary arteries, upon the muscular tissue of the heart. Hence we may exclude the notion of the first sound, at all events, being principally muscular.

Of the several theories proposed in explanation of the second ventricular sound, that of Julius Roger appears to me the simplest and the best; viz., that membranes may cause sound both by impact and by separation. The wedge-like column of blood traversing the auriculo-ventricular orifices may, hy impinging upon the separating valves, engender sound, a certain amount of force being imparted to the blood-stream, to prevent the tendency to partial vacuum left by the relaxing ventricle. Physiologists are not clear as to the actual amount of overlapping, but it is doubtless sufficient to explain the phenomenon. And one fundamental fact is admitted by most auscultators ; viz., the occasional occurrence, in the normal state, of a second sound highly intoned at the apex, while the basic sounds are weak, and, it may be, of different pitch. I shall advert to this point again, when speaking of murnurs at the apex.

The second arterial sounds are supposed to have their maxima at or near the mid sternum, and, for purposes of differential distinction, are recommended to be auscultated at the aortic and pulmonic cartilages respectively. Whether the information sought be gained by this procedure, has always appeared to me doubtful. To solve this point, I have recently attempted various experiments. The following was one. Two watches, of nearly equal timbre, intensity, and synchroneity, were covered with oilskin bags, and to the neck of each an India rubber tube was attached; the watches were placed one upon the other; and the tubes, after being connected at their origin, were made to diverge to a given distance. It was always impossible, upon listening alternately at the open end of each tube, to distingui ih the relative position of the watches, the mean note only being heard. 'I'hey were afterwards introduced into the aorta and pulmonary artery respectively of an ox recently slaughtered. Auscultation, as before, always yielded the same result. Media of different density, brought temporarily into contact with the free portion of a tube, modified the note at its open end.

It must, however, be borne in mind that synchronous with these arterial sounds, is the sudden injection of blood into the auricles from the large veins; and, from their unequivocal muscularity, it is hardly possible to conceive that this impulse can be altogether aphonous. According to a celebrated physiologist and auscultator of a certain school in Paris, Dr. Beau, although in a different serise, the auricles are said to yield under these conditions distinctly audible sound.

With respect to the second arterial sounds, it is taught by some, that the aortic sound is loudest; and this would, certainly, on à priori grounds, be probable, considering the area of the vessel and the stronger valves at its mouth. So far, however, as my own observations extend, I have not found this assertion practically true. I have observations relative to this point extending to one hundred cases, and I found on aualysis they gave the following results.

About 60 per cent. equal at both aortic and pulmonic cartilages.

, 26 " londest at aortic cartilage. $1 \pm \quad$ loudest at pulmonic cartilage. 
The question may therefore fairly be asked, whether the loudness of the second sound does stand in a direct causal relation to the strength of the ventricular systole and the reaction of the arterial walls?

I have observed in typhus fever, fatty heart, spanæmic women, and cases of general debility, that when the first sound was decidedly weak, the second was still loud and clear. In the so called accentuation of the second sound of the pulmonary artery where it would be said to exist, it has appeared to me usually rather a forcible diastolic recoil of the artery than an accent properly so called.

Taking the second aortic sound, or its replacement by murmur, as a basis for differential diagnosis, the following points have presented themselves to my notice.

A preternatural accent of the second sound in the direct course of the aorta, to the right of the sternum, has been observed and specially indicated in some cases of aortic aneurism. I have recently had an opportunity of watching throughout a case of aneurism of the ascending aorta. The earliest symptoms were those which apparently indicated an inflammatory condition of the aorta : at this period, the only persistent physical sign appreciable was marked intensification of the second sound over the aorta. Some months later, pulsating tumour was seen and felt about the second nnd third right costal cartilages; the second sound still being preternaturally loud. In the early stage, it occurred to me that the loud second sound might be explained on the hypothesis that the inflammation of the aorta had induced unusual contractility of this vessel, and thus, by rendering it more rigid, making it a better conductor of sound. At a later period, the extensive thickening of the coats, by excessive deposit, might have rendered the tube a better conductor; at all events, the intensified sound could not be explained upon the hypothesis of increased recoil of the vessel, since here the elastic reaction of the vessel was impaired or even destroyed by extreme degeneration. A further argument against the loudness of the second sound being supposed to result from the injecting force of the heart, may be borrowed from Dr. Stokes. When treating of softening of the heart in typhus fever, he states, that in his 41 st and 56th cases, the selond sound was preternaturally loud, and in the latter case attended with impulse, while the first sound was extinct, and the impulse not appreciable by palpation. I again submit, therefore, that the hypothesis of the causal relation between the loudness of the second sound and the propulsive force of the ventricle, requires reconsideration.

It is hardly necessary to mention induration of the pulmonary tissue, excavations therein, contraction of the lung from phthisis, permitting the pulmonary artery to come in immediate proximity with the chest wall-a pathologic condition first indicated, I believe, by Dr. Sibson-enlargement of the liver, etc., as sources of intensification of the sounds. I have, however, some reason to believe that a marked intensification of the sound in the post clavicular space may, now and then, assist in the diagnosis of deposit at the apices of the lungs.

In insufficiency of the aortic orifice, the second sound is more or less perfectly replaced by murmur, soft, harsh, or musical. Such murmur, however, does not nor cannot measure the amount of patency. I have particularly observed that in some cases, in which, judging from the extraordinary collapse of the arteries, the patency was extreme, that the murmur in its physical characters differed in no respect from other less advanced cases in which no collapse was observed.

I believe enlarged experience scarcely supports the notion of Dr. Hope, that aortic may be distinguished from other mur. murs by the pitch. The pitch of a murmur at any orifice infinitely varies with the physical condition engendering it. An aortic regurgitant murmur may be of so high a pitch as to approach sibilation in point of intensity.

I may add, that this distinguished observer was also decidedly in error when he stated that a mitral murmur was never audible above the level of the aortic valves. Murmurs at the orifices are not so restricted as many suppose.

There are one or two points, however, with regard to aortic diastolic murmur, which I will take leave to mention. The first is that, contrary to the opinion of some observers, I have not unfrequently found it attended with well marked diastolic fremitus even at the apex, and this in cases unattended with any excessive degree of spanæmia; and, indeed, there appears no reason why a partially rigid valve should not be susceptible of being thrown into tactile as well as sonorous vibration by he refluent current.

It is usually taught that the differential diagnosis of aortic regurgitant murmur may be, at least, in part established by the clear click of the valves of the pulmonary artery auscultated to the left. I am far from asserting this is not occasionally correct; but so far as my opportunities of observation extend, I should, in the majority of cases, deny the validity of this proposition.

When the diastolic murmur is at all intense, $I$ believe the second sound of the pulmonary artery is always more or less obscured, and in musical murmur almost invariably so. Indeed, if we bear in mind the anatomical condition existent, and consider how readily vibrations are excited in contiguous and homogeneous structures, the difficulty of conceiving the reverso is obvious.

The limits of these murmurs again, and particularly the musical, have been too much restricted; they extend over a very wide area; at times, all over the anterior and posterior surfaces of the chest, from the epigastrium to the pubis; and posteriorly, from the vertex nearly down to the lower end of the sacrum. From their site of origin, they may, I am quite sure, from comparison of cases examined after death, extend across the apex and round the axilla to the posterior scapular region; and this without coexistent disease of the mitral valve.

Excluding from consideration some rare cases of aortic aneurism, described by Gendrin as being liable to be confounded with aortic patency, is there any other condition which gives rise to similar physical phenomena? I believe there is.

A few months ago, I exhibited, at the Pathological Society, a specimen of diseased suprarenal capsules; they were removed from a female advanced in life, who had exhibited well marked symptoms of cardiac disease: a harsh double murmur was heard, with its maximum near the base, and extending with great intensity up the aortic, and downwards to the apex, axilla, and posterior scapular region. The heart's action was somewhat heaving, and its rhythm irregular. These phenomena were persistent, and scarcely underwent any change when examined on several occasions by Mr. Robinson (whose patient she was) and myself. We considered there might possibly exist both aortic and mitral disease. After death, hypertrophy of the heart was found. The aortic and mitral valves were apparently intact; but the aorta at its arch was nearly converted into a long tube; and in its ascending portion, rough, calcified portions projected, the lining membrane being entirely destroyed.

In another case, of Dr. Hawksley's, similar physical phenomena were constantly observed, and the same irregular rhythm. After death, enlargement of the heart was found; rough, calcified projections studded the ascending aorta and its arch; a slight calcification was certainly found at the base of one of the aortic valves, but not preventing tolerably fair closure; and, to the best of my judgment, quite inadequate to the production of the intense double murmur audible over base, apex, and axilla.

\section{[To be continued.]}

\section{ON THE OCCURRENCE OF AMAUROSIS IN PATIENTS SUFFERING FROM BRIGHT'S DISEASE OF THE KIDNEY.}

By Holmes Coote, F.R.C.S., Assistant-Surgeon to St. Bartholomew's Hospital, etc.

THE connexion between amblyopia, or amaurosis, and Bright's disease of the kidney, was, I believe, first pointed out in 1849 by Landouzy of Rheims, who published, in L'Union Médicale, a paper containing several cases in illustration of the fact, occurring in patients in whom nephritis had been produced by the use of alcoholic drinks, by exposure to damp, or by want of the common necessaries of life. It is a matter of surprise that, with the knowledge of the frequency with which extravasations of blood and other accidents occur within the cranium in persons the subject of albuminuria, the idea should not have more readily suggested itself of examining in such cases the condition of the nervous contents of the orbit, constituting, as they do, an integral part of the cerebro-spinal centre. We might in many instances reasonably expect to find there morbid changes of a similar character to those noticed on the convolutions, or in the substance of the brain, provided there were any means of illuminating the retina and the choroid behind the transparent media of the eye. I need scarcely remark, that this end is readily attained by the use of the ophthalmoscope.

When the kidneys are so diseased that the proper urinary 